\title{
Cronicidad: Attention and Management
}

\author{
Antonio Masiá Alegre* \\ University Expert in Management of Chronicity and Disability Care, Spain
}

Submission: January 19, 2018; Published: February 05, 2018

*Corresponding author: Antonio Masiá Alegre, University Expert in Management of Chronicity and Disability Care, Spain, Email: amasiaalegre@gmail.com

Abbreviations: CD: Chronic Diseases; PC: Primary Care; INE: National Institute of Statistics; HEC: Electronic Health Record; CCM: Chronic Care Model

\section{Opinion}

The Statistical Office of the European Union notes that in Spain in 2007, $15.7 \%$ of the population was over 65 years of age and that the forecast for the year 2050 will be $34.1 \%$, becoming one of the countries older Europeans [1]. According to data from the National Institute of Statistics (INE), due to decreases in the birth rate and mortality, in Spain 1-1-2011 there were 8 million people over 65 years old, corresponding $5.1 \%$ of the total to elderly people of 80 years or older and in this age group $5 \%$ had two or more chronic diseases (CD). However, since longevity is a determining factor in chronicity, it is not correct to associate CD with an elderly person because there are other age groups that also suffer from it [2]. The concept of the chronically ill has changed in recent times from the definition of a person affected by a single disease with more than three months duration to, at this time, lasting more than six months, with various pathologies, limiting the quality of life and important repercussions in the personal, family, psychological, social and economic. CDs surpass the health sector because they affect the quality of life and produce a great socio-economic impact and are associated with dependency and disability that cause added psycho-physical squeal. At the primary level, we must modify lifestyles and, at a secondary level, detect and treat early, in addition to rehabilitating not only the patient but also the family and their environment [3].

Primary Care (PC) regulates the flow of chronic patients and coordinates health resources and guarantees their continuity, which means caring for patients before they become so. His family and community vision is ideal to maintain a comprehensive chronic patient care. It is the continuity that allows the Family Doctor (MF) to carry out preventive activities and palliative care. Thus, the response to chronic pathology should be the reorientation of the traditional model of current curative care based on episodic contact and focused on acute processes or decomposition of the disease to a comprehensive and clinical care but also functional and social, with prevention and involvement of the patient and their environment, participating in decision-making and self-care [4]. Scientific knowledge should be appropriate for different groups of patients, from healthy people with risk factors that can make them chronic, as well as dependents and terminals. This combined vision, health and social, was launched in the United Kingdom following the Kaiser Risk stratification model. We must avoid the appearance of CD and/or the appearance of predictable complications through correct treatment and management. In this sense and as a key point we have prevention:

a) Primary: avoid the appearance of diseases acting before they appear. The medical advice on risk factors is more relevant for the promotion of health and prevention, than the screening of the risk factors of CD or the early treatment of them.

b) Secondary: Screening in healthy people that can be to the entire population or based on the active search of cases, being in the latter where the PA is the ideal framework.

c) Tertiary: acts with the disease already established, its objective being to reduce/avoid complications, delaying its evolution, avoiding disabilities and improving the quality of life.

d) Quaternary: avoid adverse effects, focusing on avoiding unnecessary health interventions or treatments for patient safety [5].

Important is also the idea of the information that the patient must have to be more active in the care of their health. For this, paternalistic attention must be abandoned in which the doctor possesses the knowledge and the patient does not. A wellinformed patient cooperates better and is more responsible, ceasing to be a passive recipient and becoming an active decisionmaker. It is a model of shared decisions where information on the part of the doctor must be truthful, based on scientific evidence, understandable, sufficient and personalized. In 
addition, responding to the complexity of chronic pathologies is difficult on the part of only a group of professionals, so teamwork (doctors, nurses, social workers...) improves functionality, reduces morbidity and mortality, decreases hospital admissions and alleviates the burden of caregivers-relatives in charge. The use of new technologies such as the Electronic Health Record (HEC) and the Internet allows information to be shared among professionals. With the first, the patient's medical information is accessed, improving care coordination and updating the therapeutic decision; while the second is an information tool for professionals and allows patients to access quality information and communication with professionals [6].

If we do a documented review on chronicity, this indicates the need to reorganize the care and strengthen the Primary Care teams (EAP), since this level of care is usually the gateway of the patient-user to the health system, where continuously and globally it is served [7]. The aging of our population is producing a progressive increase in CDs that, together with dependent people, consume $70-80 \%$ of healthcare resources. Attending to chronicity implies changes in health policies with a model of attention to chronic behaviors; for this it is necessary to carry out an integral management of chronicity in the population, coordinating patients, health professionals, caregivers and autonomic health systems. Patients come to the PC in the first instance, to hospitals for specific or urgent care and for specific treatments, therefore given that chronic patient care is the majority in this level of care, the PA should be responsible for managing and coordinating with others care levels the attention to these patients. Health reforms in the path of different crises (financial, legitimacy, rationality and quality) together with technological innovation try to present strategies and models to curb health spending [8].

The macro and meso-sanitary management (management of centers) together with micro-management (clinical management) must act on the health professional without forgetting that the community must also act, with the person being the fundamental axis. Chronicity is linked to the appearance of new models of care, reorienting the attitude of health systems towards the chronic patient:

a. Chronic Care Model.

b. Permanent Kaiser Risk Pyramid. c. Innovative Care (patients, care teams and community).

d. Priority health policies (CCAA health plans and the Plan on Strategy for the Chronicity Approach in the National Health System- SNS).

All of them mustnotonly address the economic perspective but also have to pay attention that the demographic changes (aging), the crisis and the increase of the EC need a multidisciplinary approach, in addition to acting on the outpatientization of processes and communication between the different assistance degrees [9-11]. This issue will talk about elderly people with $\mathrm{CD}$, its impact on health spending and management from the PA. In fact our health system does not work properly because it has been paying attention to acute processes and now, for a few decades, morbidity has changed quantitatively and qualitatively not serving now since most of the health resources are allocated to chronic processes.

\section{References}

1. Barrio Truchado E, Abellán García A (2006) Demographic indicators. In: The elderly in Spain. Madrid Institute for the Elderly and Social Services, Europe.

2. (2009) Ministry of Health, Social Services and Equality. European Health Survey in Spain, Europe.

3. Contel JC (2011) Care for the chronic patient in a situation of complexity: the challenge of building integrated care. Primary Care 2011.

4. Ruiz Azarola A, Parestelo Pérez L. Citizen participation in health: training and shared decision-making. SESPAS Report 2012. Gac.Sanit. 2012

5. (2002) WHO. Innovative care for chronic conditions.

6. (2010) Strategy to address the challenge of chronicity in Euskadi. Basque Government, Spain.

7. (2012) Strategy for dealing with chronicity in the National Health System (NHS). Ministry of Health, Social Services and Equality.

8. Bengoa R, Nuño R (2008) Heal and care Innovation in the management of chronic diseases: Barcelona. Elssevier-Masson.

9. Nuño R (2010) Improvement of Primary Care with chronic conditions The chronic care model. Question of models? Keys to a debate. Report of the Spanish Society of Primary Care, Saned Group p. 86-93.

10. Fernández J (2006) Medicine based on obedience. ABC of Health Management.

11. Bodenheimer T, Naguer EH, Grumbach K (2002) Improving primary care for patients with chronic illiness. JAMA 288(14): 1775-1779.

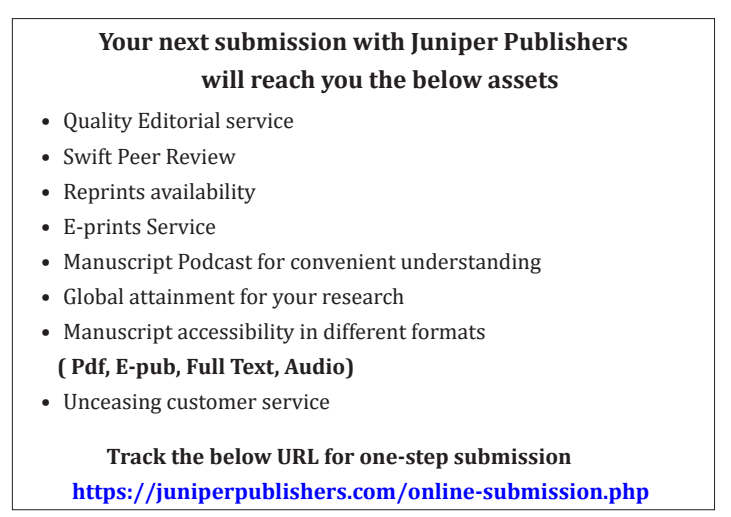

